\title{
Interval Between the Synthesis and Assembly of Cytoskeletal Proteins in Cultured Neurons
}

\author{
Mark M. Black, ${ }^{*}$ Patricia Keyser, ${ }^{*}$ and Eugene Sobel $\dagger$ \\ Departments of *Anatomy and †Family Practice and Community Health, Temple University School of Medicine, \\ Philadelphia, Pennsylvania 19140
}

We have used pulse-chase experiments to study the time interval between the synthesis and assembly of tubulin and neurofilament proteins (NFP) in sympathetic neurons grown in tissue culture. After varying pulse-chase times, cultures were extracted with Triton $X-100$ such that polymerized tubulin and NFP were insoluble, while unassembled tubulin and NFP were quantitatively solubilized. The partitioning of labeled tubulin and NFP between Triton X-100-soluble and insoluble, or cytoskeletal, fractions was determined with an isoelectric focusing $\times$ SDS gel electrophoresis assay. Labeled tubulin and NFP in cultures pulse-labeled for 5-10 min partitions primarily with the soluble fraction. When pulse-labeled cultures were chased for increasing periods of time, relatively more of the total labeled tubulin and NFP partitioned with the cytoskeleton, attaining maximal values after chase times of 60-120 and $15-30 \mathrm{~min}$, respectively. The maximal values for the relative levels of labeled tubulin and NFP in polymer were $70-75$ and $>90 \%$, respectively. The levels of labeled tubulin and NFP synthesized during a short pulse-label remained constant for at least $2 \mathrm{hr}$, indicating that selective turnover of soluble tubulin and NFP does not detectably contribute to the changes in solubility properties of these proteins observed in the pulse-chase experiments. These results indicate that newly synthesized tubulin and NFP are rapidly assembled from soluble precursors. The lag between the synthesis and assembly of the 145,000 -molecular-weight NFP is not related to its phosphorylation because its initial incorporation into the cytoskeleton occurs prior to its phosphorylation. The relatively short time interval between the synthesis and assembly of NFP and tubulin suggests that the distance between their sites of synthesis and assembly is relatively small, and thus supports the hypothesis that newly synthesized tubulin and NFP are assembled in relatively close proximity to their sites of synthesis, which are localized primarily in the neuron cell body. If this is correct, then these cytoskeletal proteins are assembled in the soma prior to their entry into the neurites.

The axonal and dendritic cytoskeletons consist principally of microtubules, microfilaments, and neurofilaments (Peters et al., 1976). These structures are organized into interacting networks that extend from the cell body to the tips of their axonal and dendritic neurites. The axonal and dendritic cytoskeletons provide the structural framework that defines the external shape of these neurites. Thus, identifying how the structure of the axonal

\footnotetext{
Received July 2, 1985; revised Sept. 16, 1985; accepted Oct. 9, 1985.

We are grateful to Drs. Raymond Lasek and Michael Katz for their comments on the manuscript and to Drs. Lucy Autillo-Gambetti, Ronald Liem, and Virginia Lee for providing us with antibodies against the 68,000 neurofilament subunit. The work reported here was supported by NIH Grant NS17681 to M.M.B. Dr. Black is also the recipient of a Research Career Development Award from NIH.

Correspondence should be addressed to Dr. Black, Department of Anatomy, Temple University School of Medicine, 3420 North Broad Street, Philadelphia, PA 19140.

Copyright (C) 1986 Society for Neuroscience $0270-6474 / 86 / 041004-09 \$ 02.00 / 0$
}

and dendritic cytoskeletons is produced and maintained is central to studies of the development of neuronal morphology.

One important question concerning the axonal and dendritic cytoskeletons is "Where are the axonal and dendritic cytoskeletons initially assembled?" One hypothesis, which is based on axonal transport studies (Black and Lasek, 1980; Lasek, 1981), holds that the proteins comprising these cytoskeletons are assembled in close proximity to their sites of synthesis. These proteins are synthesized in the neuron cell body. Thus, the axonal and possibly dendritic cytoskeletons may be initially assembled in the neuron cell body. Support for this possibility stems from morphological studies that show that networks of neurofilaments and microtubules that are characteristic of neurites are present in the neuron soma and appear to be continuous into the neurites (Lasek and Brady, 1982; Smith, 1973; Weiss and Wang, 1936).

In the present report, we have studied the time interval between the synthesis of cytoskeletal proteins and their incorporation into the cytoskeleton in order to obtain information on the spatial relationship between the sites for the synthesis and assembly of these proteins. These studies have focused on tubulin and neurofilament proteins (NFP). Portions of the results presented here have been published previously in abstract form (Black and Kurdyla, 1983b).

\section{Materials and Methods}

\section{Cell culture}

Superior cervical ganglia of 1- to 5-d-old rat pups were used as a source of sympathetic neurons. Neurons were dissociated by sequential treatment with collagenase and then trypsin as described in Black and Kurdyla (1983a), and then plated onto collagen-coated culture dishes. Culture conditions were as described previously (Peng et al., 1985). Cultures were freed of nonneuronal cells by treatment with antimitotic poisons and used at 1-2 weeks of age.

\section{Metabolic labeling}

For most experiments, cells were fed with medium preequilibrated to $36^{\circ} \mathrm{C}$ and $\mathrm{pH} 7.3$ and containing $\sim 5 \%$ of the normal level of methionine for 30-60 min prior to the addition of ${ }^{35} \mathrm{~S}$-methionine. In experiments on tubulin, cultures were labeled with $100 \mu \mathrm{Ci}$ of ${ }^{35} \mathrm{~S}$-methionine, while in experiments on NFP, cultures were labeled with 250-1000 $\mu \mathrm{Ci}$. In experiments on NFP, the isotope was lyophilized, dissolved in a small volume of medium, and then added to the cultures. Cultures were incubated with label for 5-15 min (as specified in the text) and then processed as described below, or chased for varying times prior to analysis. The chase period was initiated by replacing the labeled medium with unlabeled medium, equilibrated to appropriate temperature and $\mathrm{pH}$, and containing $10 \times$ the normal levels of unlabeled methionine.

\section{Cell extraction}

To separate assembled from unassembled forms of tubulin and NFP, cultures were extracted with the nonionic detergent Triton X-100 under 
Table 1. Percentage of labeled tubulin in polymer

\begin{tabular}{llllll}
$\begin{array}{l}\text { Labeling } \\
\text { time }\end{array}$ & With glycerol & & \multicolumn{2}{l}{ Without glycerol } \\
\cline { 2 - 3 } \cline { 5 - 6 } & Without taxol & With taxol & & Without taxol With taxol \\
\hline $10 \mathrm{~min}$ & $29 \pm 2(n=9)$ & $36(n=2)$ & & $16(n=2)$ & $38(n=2)$ \\
$16 \mathrm{hr}$ & $78 \pm 2(n=6)$ & $86(n=2)$ & $60(n=2)$ & $88(n=2)$ \\
\hline
\end{tabular}

Cultures labeled for the indicated times were extracted as described under Materials and Methods using the buffers described therein (with glycerol) or in Schliwa et al. (1981). (These latter buffers contained $0.2 \%$ Triton X-100, but no glycerol [Without glycerol].) The resulting soluble and cytoskeletal fractions were analyzed on 2-D gels and the radioactivity associated with tubulin was quantified as described in Materials and Methods. The data shown are averages of two or more determinations; SE are also shown for the data based on more than two determinations.

solution conditions that stabilized and thereby rendered insoluble assembled tubulin or NFP, and also quantitatively solubilized unassembled forms of these proteins. The partitioning of tubulin or NFP between the soluble and insoluble fractions was then analyzed by isoelectric focusing $\times$ SDS (2-D) gel electrophoresis, immunoblotting, or immunoprecipitation. The specific solution conditions used to study tubulin and NFP are described below.

\section{Tubulin}

Cultures were rinsed twice with PBS and once with buffer $1(0.1 \mathrm{M}$ PIPES, pH 6.9, 2 M glycerol, $1 \mathrm{~mm} \mathrm{MgSO}_{4}, 2 \mathrm{~mm}$ EGTA and a cocktail of protease inhibitors [0.2-1 trypsin inhibitory units/ml of approtinin, and $10 \mu \mathrm{g} / \mathrm{ml}$ of leupeptin, chymostatin, and antipain]). The cells were then lysed while remaining attached to the dish in buffer $1+0.2 \%$ wt/ vol Triton X-100 by incubation for two successive 10 min periods with fresh buffer. The resulting Triton X-100-soluble material, which contained unassembled tubulin, was pooled. The Triton X-100-insoluble residue, or cytoskeleton, was rinsed twice with buffer 1 without EGTA and then dissolved in sample buffer ( $8 \mathrm{~m}$ urea, 2\% SDS, and 5\% 2 -mercaptoethanol). The above-described procedures are modified from our previous work (Black et al., 1984) and have been shown to effectively stabilize microtubules and to quantitatively separate unassembled from assembled microtubule proteins.

\section{NFP}

Cultures were rinsed twice with PBS and then lysed by incubation in a buffer containing $0.1 \mathrm{M}$ PIPES, pH 6.9, $1 \% \mathrm{wt} / \mathrm{vol}$ Triton X-100, $1 \mathrm{~mm}$ $\mathrm{MgSO}_{4}, 5$ mM EGTA, $0.19 \mathrm{M} \mathrm{NaCl}$, and protease inhibitors for two successive 5 min periods with fresh buffer. The resulting Triton X-100soluble material was pooled and then centrifuged at $12,000 \times g$ for 10 $\min \left(a t 4^{\circ} \mathrm{C}\right)$ to pellet any cytoskeletal material that may have detached from the dish during extraction. The supernate was used for subsequent analyses (see below). The Triton X-100-insoluble cytoskeleton was rinsed twice with fresh buffer and then dissolved in either sample buffer (see above) or $1 \% \mathrm{wt} / \mathrm{vol}$ SDS with protease inhibitors.

\section{2-D gels}

In most experiments, Triton X-100-soluble and cytoskeletal fractions were analyzed directly on 2-D gels. 2-D gel electrophoresis was performed as previously described (Black et al., 1984) and labeled proteins were visualized by fluorography (Bonner and Laskey, 1974). Triton $\mathrm{X}-100$-soluble fractions were precipitated with methanol and then dissolved in sample buffer. The partitioning of tubulin and NFP between soluble and cytoskeletal fractions was quantified by excising appropriate spots from the 2-D gels, dissolving them in $\mathrm{H}_{2} \mathrm{O}_{2}$ and then quantifying their ${ }^{35} \mathrm{~S}$-content by scintillation counting. The relative levels of labeled tubulin or NFP in polymer were calculated as

\section{CPM cytoskeletal tubulin or NFP \\ $\overline{\mathrm{CPM} \text { soluble }+ \text { cytoskeletal tubulin or NFP }} \times 100$}

\section{Immunoprecipitation and immunoblotting}

In some experiments, the partitioning of NFP between soluble and cytoskeletal fractions was evaluated by immunoprecipitation or immunoblotting, using one of the following antibodies: a polyclonal antibody to the 68,000 NFP (NF68) (obtained from Dr. L. Autillo-Gambetti,

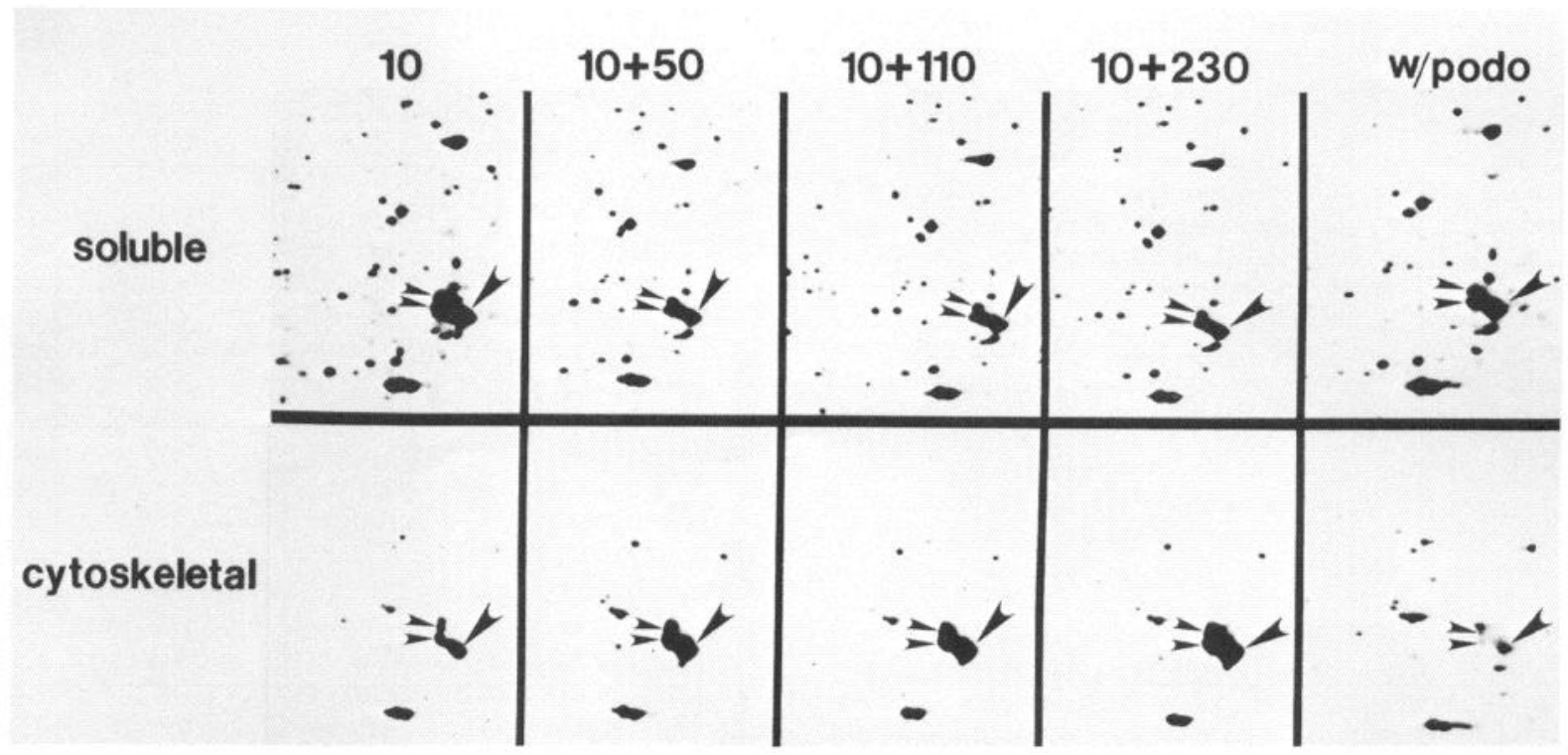

Figure 1. Pulse-chase experiments on the partitioning of newly synthesized tubulin between soluble and cytoskeletal fractions. Cultures labeled with ${ }^{35} \mathrm{~S}$-methionine for $10 \mathrm{~min}$ were either extracted with the Triton X-100-containing microtubule stabilizing buffer as described in Materials and Methods or chased in the presence of excess unlabeled methionine for 50,110, or 230 min prior to extraction. The resulting soluble and cytoskeletal fractions, which contain unassembled or assembled tubulin, respectively, were analyzed on 2-D gels, and labeled proteins were visualized by fluorography. The figure shows portions of fluorographs that depict the labeled proteins in soluble (upper panels) and cytoskeletal (lower panels) fractions. The labeling conditions are indicated above each pair of fluorographs. Four tubulin subunits can be resolved in the gels, two $\alpha$-tubulins (small arrowheads) and two $\beta$-tubulins, of which one is particularly prominent and is identified by large arrowheads. After a 10 min label, the majority of labeled tubulin partitions with the soluble fraction, and, with increasing chase times, an increasingly greater proportion of the labeled tubulin partitions with the cytoskeletal fraction. The panels on the far right (w/PODO) show portions of fluorographs of soluble and cytoskeletal fractions obtained from a culture labeled for $24 \mathrm{hr}$ and also treated with podophyllotoxin, a microtubule depolymerizing drug, for several hours prior to extraction. Note that the vast majority of labeled tubulin partitions with the soluble fraction. 


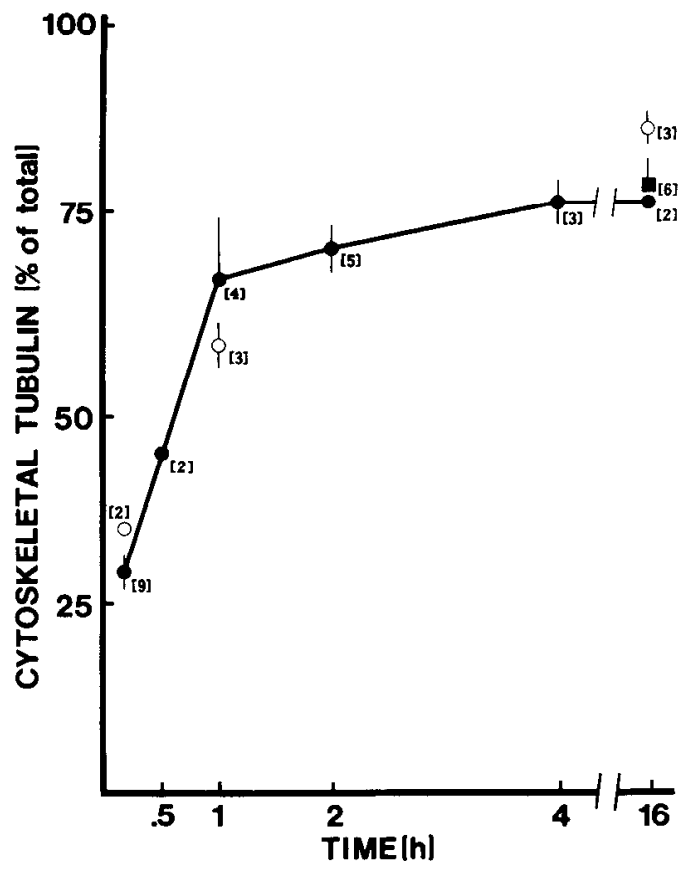

Figure 2. Quantitative analysis of the partitioning of tubulin between soluble and cytoskeletal fractions. Triton X-100-soluble and -insoluble fractions were prepared from cultures labeled for $10 \mathrm{~min}$ and then chased for times ranging from 0 to $16 \mathrm{hr}$ and analyzed on 2-D gels. The tubulin spots were excised from the gels and their radioactivity quantified as described in Materials and Methods. The percentage of total labeled tubulin associated with the cytoskeleton was calculated as described under Materials and Methods and then graphed as function of time. Data shown are means $\pm \mathrm{SE} ; n$ is indicated in brackets. Closed circles depict data obtained with the standard extraction procedure as described under Materials and Methods. Open circles show data obtained from cultures extracted in the presence of $60 \mu \mathrm{m}$ taxol and glycerol. Filled square shows the data obtained from cultures labeled continuously for 16-20 hr.

Department of Pathology, Case Western Reserve University, Cleveland, $\mathrm{OH}$ ), a polyclonal antibody against the neurofilament triplet (obtained from Dr. R. K. H. Liem, Department of Pharmacology, New York University School of Medicine, New York, NY), a monoclonal antibody against NF68 (from Dr. V. Lee, Department of Neuropathology, University of Pennsylvania, Philadelphia, PA), and monoclonal antibodies that recognized either highly phosphorylated or nonphosphorylated forms of the 145,000 and 200,000 NFP (NF145 and NF200, respectively) (from Sternberger-Meyer Immunocytochemicals). The antibody to nonphosphorylated NFP also recognizes poorly phosphorylated forms of the NF200 (M. M. Black, unpublished observations). Immunoblotting and immunoprecipitation procedures were performed as described (Peng et al., 1985).

\section{Peptide mapping}

One-dimensional (1-D) peptide mapping was performed as described by Black et al. (1984) using 50 or $150 \mathrm{ng}$ of Staphylococcus aureus V8 protease per sample. All proteins for peptide mapping were excised from 2-D gels.

\section{Results}

\section{Stabilization of polymerized tubulin and NFP and} separation of unpolymerized and polymerized tubulin and NFP

We have coupled pulse-chase experiments and extraction with Triton X-100 to study the time interval between the synthesis and assembly of cytoskeletal proteins. Correct interpretation of the results depends on the ability of the extraction procedures to effectively stabilize polymerized tubulin and NFP, and to separate unpolymerized from polymerized forms of these proteins. Several experiments have been carried out to evaluate the extraction procedures used in our studies.

\section{Experiments on tubulin}

The extraction procedures used to separate unpolymerized and polymerized tubulin rely on their differential solubility in a Triton X-100-containing microtubule stabilizing buffer. With the specific solution conditions used, polymerized tubulin is insoluble, while unpolymerized tubulin is soluble. To determine the effectiveness with which unpolymerized tubulin is solubilized during extraction, cultures labeled with ${ }^{35} \mathrm{~S}$-methionine for 24 $\mathrm{hr}$ were treated with or without the microtubule-depolymerizing drug podophyllotoxin prior to extraction. Electron microscopy showed that microtubule levels were greatly reduced in drugtreated cultures compared to controls, although a few microtubules were present in the drug-treated cultures (not shown). Biochemical analyses showed that $\sim 78 \%$ of the tubulin in control cultures partitioned with the cyloskeletal fraction (Table 1). In contrast, the vast majority of the labeled tubulin in the drugtreated cultures partitioned with the soluble fraction (Fig. 1, w/PODO). Similar results were also obtained when cultures were extracted with Triton X-100 in the presence of 1 or $5 \mathrm{~mm}$ $\mathrm{CaCl}_{2}$ (Black et al., 1984). The low levels of cytoskeletal tubulin in drug-treated cultures are assembled into stable microtubules, which in cultured sympathetic neurons contain $\sim 5 \%$ of the total tubulin (Black et al., 1984). These results show that the extraction conditions effectively solubilize unpolymerized tubulin and that the tubulin partitioning with the cytoskeletal fraction is in a polymeric form that corresponds primarily to microtubules (see also Black et al., 1984).

To evaluate the fidelity of microtubule preservation during extraction, we compared the relative levels of polymerized tubulin in cultures extracted in the presence or absence of $60 \mu \mathrm{M}$ taxol. Taxol is a potent microtubule stabilizer that rapidly binds to microtubules and thereby prevents their depolymerization (Morris and Lasek, 1984; Schiff et al., 1979). As seen in Figure 2 and Table 1 , the relative levels of polymerized tubulin obtaincd from culturcs lysed with glycerol-containing buffers in the presence of taxol closely resemble the values obtained from cultures extracted without taxol. We have also examined the effects of taxol on the partitioning of tubulin during extraction with buffers that lack glycerol. In the absence of taxol, the relative levels of polymerized tubulin obtained with glycerol-free buffers were substantially less than the values obtained with glycerol-containing buffers (Table 1). When taxol is included in buffers without glycerol the relative levels of labeled tubulin recovered as polymer are substantially higher, and closely resemble the levels obtained with glycerol-containing buffers (Table 1). We previously showed that the glycerol-containing buffers induce little if any tubulin polymerization during extraction (Black et al., 1984). These results show that the presence of glycerol in the extraction buffers substantially reduces the extent of microtubule depolymerization that occurs during extraction, and that the addition of taxol to suboptimal extraction buffers effectively eliminates most and possibly all of the depolymerization that occurs in these buffers. Thus, the minimal effect of taxol on the recovery of polymerized tubulin during extraction with glycerol-containing buffers indicates that these buffers faithfully preserve microtubules.

\section{Experiments on $N F P$}

Immunoblot analyses show that cultured sympathetic neurons contain the neurofilament triplet (Fig. 3). Some immunoblots were obtained with antibodies that recognized phosphorylated or unphosphorylated forms of the NF145 and NF200, and as shown in Figure $3, b$ and $c$, these antibodies recognized distinct sets of proteins (see also Fig. 7). Antibody against unphosphor- 


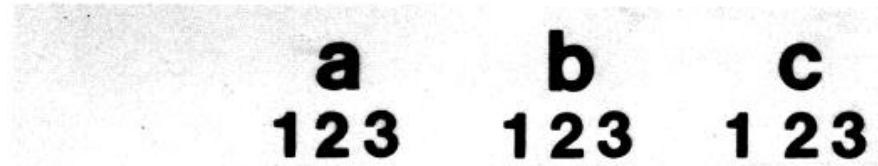

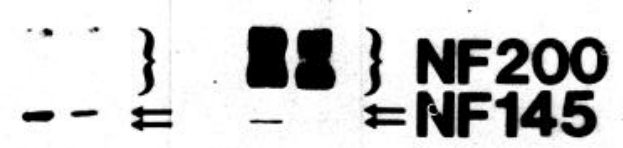

Figure 3. Immunoblot analyses of NFP in soluble, cytoskeletal, and SDS extracts of cultured sympathetic neurons. Nitrocellulose transfers were probed with monoclonal antibodies to NF68 (a), phosphorylated NF145 + NF200 $(b)$, or nonphosphorylated NF145 + NF200 (c). For each nitrocellulose transfer, lanes 1 and 2 contain the soluble and cytoskeletal fractions, respectively, prepared from an individual culture, and lane 3 contains an SDS extract from a different culture. Each of the NFP partitions quantitatively with the cytoskeletal fraction (compare lanes 1 and 2 in each panel). Note also that the pattern of NFP in the cytoskeletal fractions is indistinguishable from that in the SDS extract (compare lanes 2 and 3 in each panel), indicating that there is negligible degradation or dephosphorylation of NFP during extraction. ylated NFP recognized a single variant of NF145 and several species of NF200 that ran as a broad band in 1-D gels (Fig. 3) or a series of spots in 2-D gels (Fig. 7) that ranged in pI from 5.5 to 6.5 and in apparent molecular weight from 165,000 to 190,000 . The antibody against phosphorylated NFP recognized three distinct species of NF145 and trace amounts of NF200 (Figs. $3 b$ and $7 d$ ). The antibodies to phosphorylated and nonphosphorylated NFP recognize distinct forms of NF145 (see Fig. 7). A more detailed account of the subunit composition of intermediate filament proteins of cultured sympathetic neurons and their posttranslational modifications will be presented in a separate manuscript (M. M. Black and P. Keyser, unpublished observations).

Neurofilaments, like intermediate filaments in nonneuronal cells (Blikstad and Lazarides, 1983), remain intact following extraction with fairly harsh buffers (Drake and Lasek, 1984; Morris and Lasek, 1982). Accordingly, we have used a fairly harsh extraction procedure to separate unpolymerized NFP from polymerized NFP (see Materials and Methods), and with this procedure, all NFP, including the NF200 species recognized by the antibody against nonphosphorylated forms of NFP, partitioned almost exclusively with the cytoskeletal fraction (Fig. 3). Trace levels of soluble NFP could be detected, but only with heavily overloaded gels probed with relatively high concentrations of antibody (not shown). These results show that NFP in cultured sympathetic neurons are almost entirely polymerized. Electron-microscopic analyses indicate that NFP in the cytoskeletons correspond primarily to neurofilaments (Black et al., 1984; Drake and Lasek, 1984; Morris and Lasek, 1982).

\section{Time interval between the synthesis and assembly of tubulin}

Figures 1 and 2 show kinetic analyses of the changes in relative levels of labeled tubulin that were polymerized in cultures labeled for $10 \mathrm{~min}$ and then chased for times between 0 and 16 hr. In cultures labeled for $10 \mathrm{~min}, \sim 30 \%$ of the labeled tubulin was polymerized. This value increased steadily to $70-75 \%$ when pulse-labeled cultures were chased for $1-2 \mathrm{hr}$, and this value was then maintained for at least $16 \mathrm{hr}$. Some $70-75 \%$ of the labeled tubulin was also polymerized in cultures labeled continuously for $16 \mathrm{hr}$ (Table 1) or $72 \mathrm{hr}$ (not shown). These and other observations (Black et al., 1984) indicate that $\sim 75 \%$ is the steady-state value for the relative level of total tubulin that is assembled into microtubules. To summarize, newly synthesized tubulin is assembled from soluble precursors, reaching the apparent in vivo steady state within 1-2 hr after its synthesis.

We have examined tubulin turnover to evaluate whether selective degradation of soluble tubulin contributes to the changes in relative levels of polymerized tubulin observed in the pulsechase experiments. The results indicate that the levels of labeled tubulin synthesized during the pulse period did not detectably decay during a chase period of $2 \mathrm{hr}$ (not shown). Thus, the changes in relative levels of polymerized tubulin observed in the pulse-chase experiments reflect the dynamic interactions between assembled and unassembled tubulin, and not selective turnover of unpolymerized tubulin.

\section{Time interval between the synthesis and assembly of NFP}

Although the vast majority of NFP in cultured sympathetic neurons is polymerized (Fig. 3), newly synthesized NFP is in great part unpolymerized (Fig. 4, Table 2). In one series of experiments, soluble and cytoskeletal fractions from a culture pulse-labeled for 7.5 min were probed for NF68 by immunoprecipitation (Fig. $4 c$ ). In these experiments, labeled NF68 partitioned largely with the soluble fraction, although it was also present in the cytoskeletal fraction. Comparable results for NF68 and also for NF145 were obtained when soluble and cytoskeletal fractions were analyzed directly on 2-D gels (Fig. 4, $a$ and $b$ ). Note also that with short pulse times, relatively more of the 


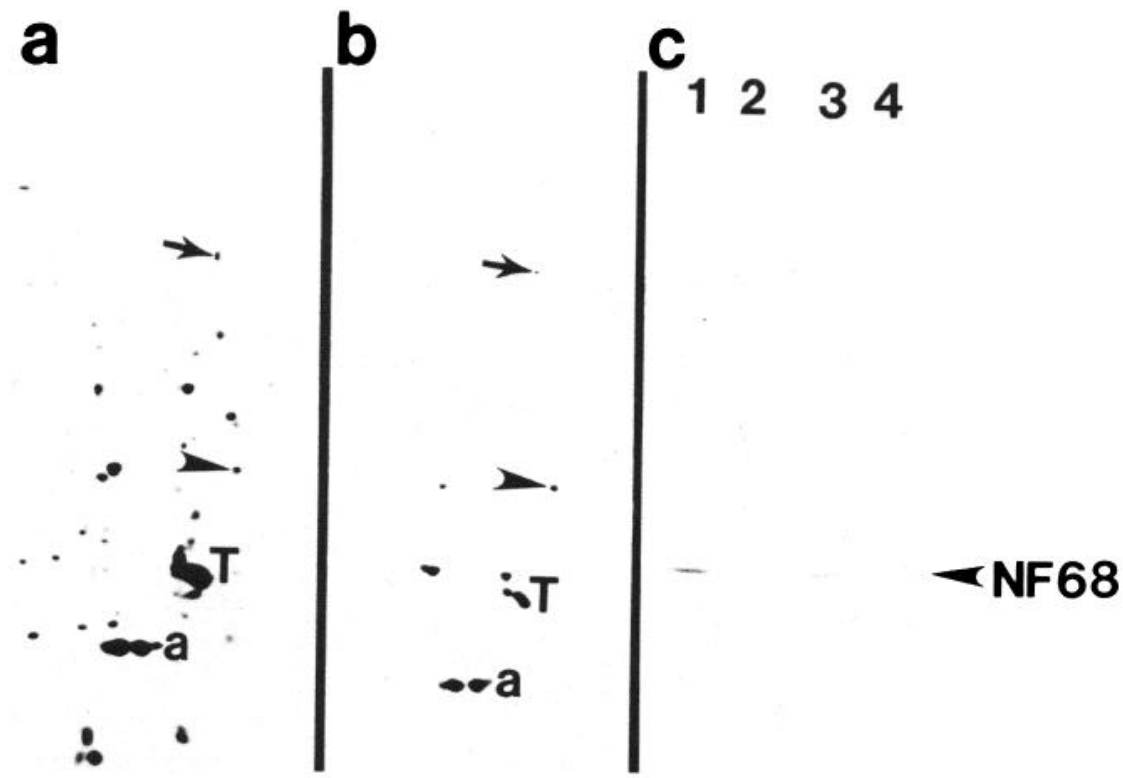

Figure 4. 2-D gel and immunoprecipitation analyses of the solubility of newly synthesized NFP. $a$ and $b$, Portions of fluorographs depicting the labeled proteins in the soluble and cytoskeletal fractions, respectively, prepared from a culture pulse-labeled for 5 min. The NF68 and NF145 are indicated by arrowheads and arrows, respectively. One-dimensional peptide mapping has established that the designated proteins in the soluble fraction are indistinguishable from their counterparts in the cytoskeletal fraction (data not shown). Moreover, the identity of the NF68 and NF145 in the 2-D gels is based on comigration with protein spots recognized by antibodies to the NF68 and nonphosphorylated forms of the NF145, respectively. To carry out these analyses, soluble and cytoskeletal fractions from pulse-labeled cultures were supplemented with unlabeled cytoskeletal material, analyzed on 2-D gels, transferred to nitrocellulose, and then blotted with antibodies against NF68 or nonphosphorylated NFP. Autoradiographs were prepared from the immunoblots and the labeled proteins that comigrated with the immunoreactive species were identified. These experiments allowed unequivocal identification of the NF68 and NF145 in 2-D gels, but not the NF200. $T$, tubulin; $a$, actin. Note that the majority of the labeled NF68 and NF145 partitions with the soluble fraction. $c$, Soluble and cytoskeletal fractions obtained from cultures labeled for 7.5 min were probed for the NF68 by immunoprecipitation with a polyclonal antibody to the NF68. Lanes 1 and 2 show the material precipitated from the soluble fraction by the antibody or a nonimmune IgG, respectively, while lanes 3 and 4 show the labeled material precipitated from the cytoskeletal fraction by the antibody or nonimmune IgG, respectively. Only the upper two-thirds of the fluorograph is shown. The position of the NF68 is indicated by an arrowhead.

labeled NF68 appeared to partition witih the cytoskeletal fraction than did the labeled NF145 (see Figs. 4 and 5, Table 2). The solubility properties of newly synthesized NF200 have not been evaluated because we could not definitively identify labeled NF200 spots in the 2-D gels.

To determine whether the labeled NF68 and NF145 in the soluble fraction were truly unpolymerized, rather than polymerized material that detached from the culture dish during Triton X-100 extraction, Triton X-100-soluble fractions from cultures labeled for $15 \mathrm{~min}$ were centrifuged at $180,000 \times g$ for $30 \mathrm{~min}$, and the resulting supernates and pellets were analyzed on 2-D gels. The labeled NF68 and NF145 were quantitatively recovered in the supernate (not shown), indicating that these proteins were in monomeric and/or small oligomeric forms.

Pulse-chase experiments were carried out to determine the time interval between the synthesis and assembly of NF68 and

\begin{tabular}{lll}
\hline Table 2. Percentage of labeled NFP in polymer & \\
Labeling time & NF68 & NF145 \\
\hline 15 min $(n=2)$ & 66 & 43 \\
15 min with a 15 min chase $(n=2)$ & 86 & 89 \\
$16 \mathrm{hr}(n=3)$ & $94 \pm 2$ & $91 \pm 3$ \\
\hline
\end{tabular}

Cultures labeled as described above were extracted as described in Materials and Methods. The fractions were analyzed on 2-D gels and the radioactivity associated with the NF68 and NF145 was quantified as described in Materials and Methods. The data shown are averages of two or three determinations; SE are shown for the $16 \mathrm{hr}$ samples.
NF145 (Fig. 5, Table 2). In pulse-labeled cultures, substantial levels of labeled NFP were unpolymerized (Figs. 4 and 5, Table 2 ). When such cultures were chased in the absence of label, the relative levels of labeled NFP in polymer rapidly increased, reaching values of $\sim 90 \%$ with chase times as short as $15 \mathrm{~min}$ (Table 2). With chase times of $2 \mathrm{hr}$ or longer, labeled NFP were only detected in the cytoskeletal fraction.

The levels of radioactivity associated with NF68 and NF145 in cultures pulse-labeled for $15 \mathrm{~min}$ remained constant for at least $2 \mathrm{hr}$ (data not shown). Thus, the increase in the relative levels of labeled NFP in polymer observed in the pulse-chase experiments reflects a shift of these proteins from monomer into polymer, and not selective degradation of monomer.

\section{Polymerization of the NF145 is not coupled to its phosphorylation}

NF145 of brain undergoes extensive phosphorylation (Julien and Mushynski, 1982), and it has been suggested that the polymerization of NF145 may be coupled to its phosphorylation (Wong et al., 1984). NF145 of cultured sympathetic neurons is posttranslationally modified, and aspects of this can be seen in the pulse-chase experiments of Figure 5 , in which several variant forms of the NF145 appear in pulse-labeled cultures chased for prolonged times. These variant forms are generated in part by differential phosphorylation (M. M. Black and P. Keyser, unpublished observations). Modified forms of newly synthesized NF145 first become detectable in cytoskeletons (Fig. 6), as well as in whole-cell extracts (not shown), 30-60 min after the protein is synthesized (Fig. 6). However, newly synthesized NF145 becomes polymerized within minutes of its synthesis (Figs. 4 and 


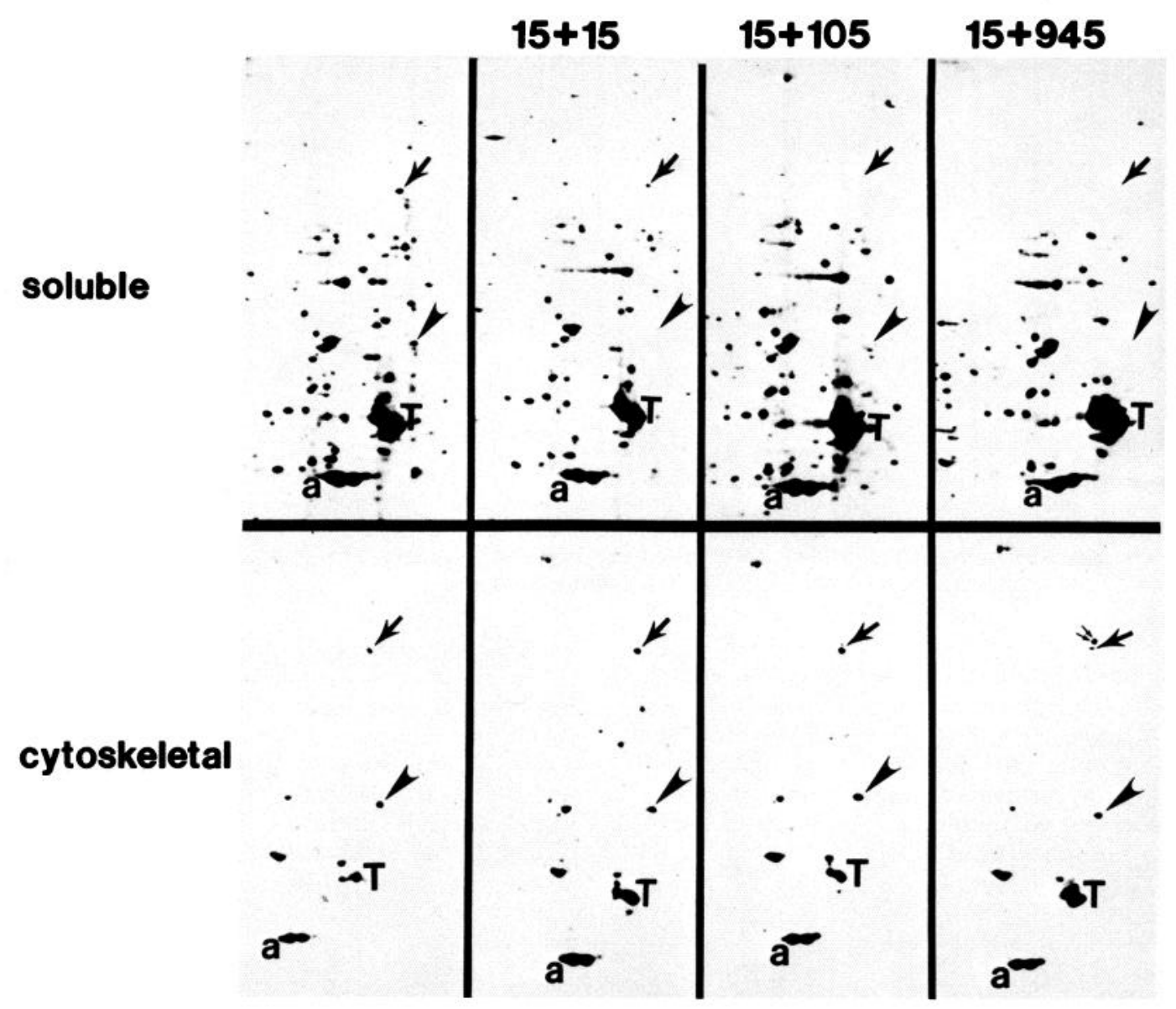

Figure 5. Pulse-chase experiments on the partitioning of newly synthesized NF68 and NF145 between soluble and cytoskeletal fractions. The figure shows portions of fluorographs of 2-D gels of soluble (upper panels) and cytoskeletal (lower panels) fractions prepared from cultures pulselabeled for $15 \mathrm{~min}$ and then chased for times ranging from 0 to $945 \mathrm{~min}(16 \mathrm{hr})$. The specific pulse-chase conditions are indicated above each pair of fluorographs. The arrowheads identify the position of the NF68, while the arrows identify the position of the NF145, specifically the species that migrates fastest in the SDS dimension. In the cytoskeletal fraction obtained from the culture chased for 945 min, several variants of the NF145 are indicated with arrows. The large arrow identifies the fastest migrating variant, while the small arrows identify the additional variants that appear with prolonged chase times. Note that at the end of the $15 \mathrm{~min}$ pulse, labeled NF68 and NF145 are roughly equally distributed between the soluble fractions and cytoskeletal fractions, and that the partitioning of these proteins shifts almost entirely to the cytoskeletal fraction during the chase period. In fact, this shift is almost complete with chase times as short as $15 \mathrm{~min}$.

$5)$. These results suggest that newly synthesized NF145 is incorporated into the cytoskeleton prior to undergoing posttranslational modifications such as phosphorylation. This possibility is further supported by the observation that the vast majority of the NF145 detectable with antibody to nonphosphorylated NFP partitions with the cytoskeleton (Fig. 3) (see also Bennett and DiLullo, 1985). Also, newly synthesized and assembled NF145 comigrated precisely in 2-D gels with the NF145 variant recognized by antibody against nonphosphorylated NFP, but not with the NF145 species recognized by antibody against phosphorylated NFP (Fig. 7). We conclude that in cultured sympathetic neurons the majority of the NF145 is incorporated into the cytoskeleton prior to its phosphorylation.

\section{Discussion}

Tubulin and NFP are rapidly assembled from soluble precursors

We have used pulse-chase experiments to study the time interval between the synthesis and assembly of tubulin and NFP in cultured neurons. The results can be summarized as follows. First, labeled tubulin and NFP synthesized during short pulselabels partition primarily with the soluble fraction during ex- traction with Triton $\mathrm{X}-100$ under solution conditions that stabilize and thereby render insoluble polymerized forms of these proteins (Figs. 2 and 4, Tables 1 and 2). Second, soluble NFP remain in solution even after centrifugation at high centrifugal forces, indicating that they are in a monomeric or possibly short oligomeric form, rather than short neurofilaments. Comparable results have also been obtained for soluble tubulin (Black et al., 1984). Third, newly synthesized monomeric (or protomeric) NFP or tubulin could be chased into the cytoskeletal fraction (Figs. 1, 2, and 5). These results demonstrate that newly synthesized tubulin and NFP are assembled from soluble precursors.

Newly synthesized tubulin and NFP are polymerized relatively rapidly. The pulse-chase experiments show that these proteins appear in a polymerized form within minutes of their synthesis, and that by $60-120 \mathrm{~min}$ (tubulin) and $30 \mathrm{~min}$ (NFP) after their synthesis, they have reached the apparent intracellular steady-state monomer-polymer distribution (Figs. 2 and 5, Table 2). The lag observed between the synthesis and assembly of tubulin and NFP may reflect several factors, including diffusion from the site(s) of synthesis to the site(s) of assembly and the dynamics of the intracellular equilibria for the assembly of these proteins into their respective polymers. 


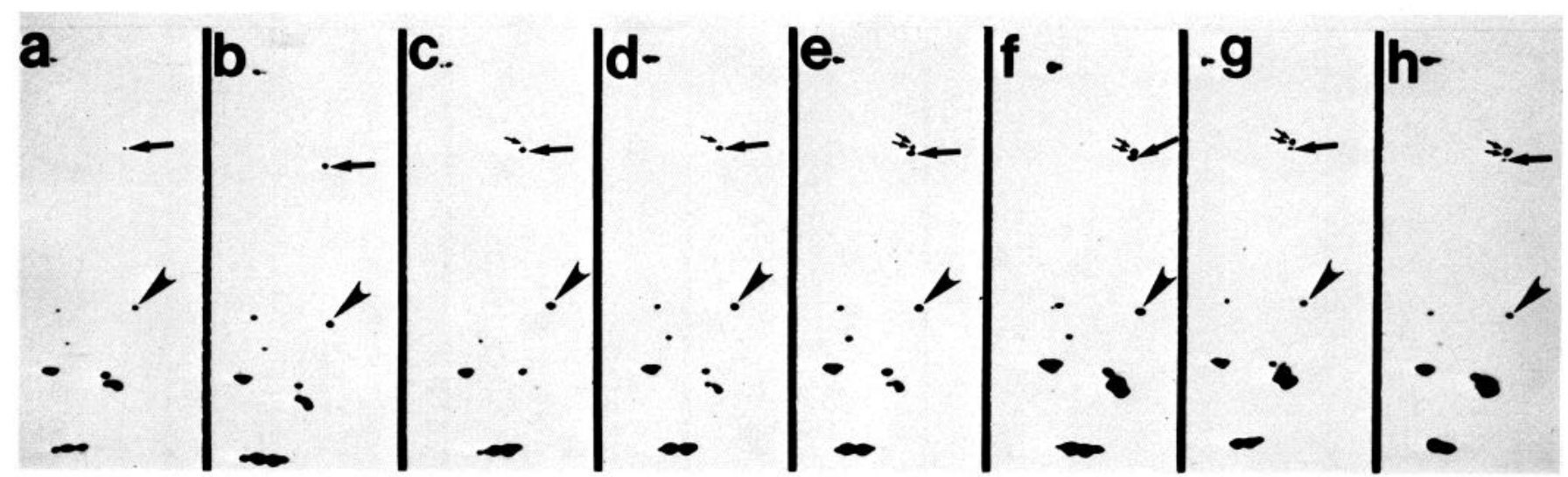

Figure 6. Pulse-chase experiments on the 2-D gel appearance of the NF68 and NF145. The figure shows fluorographs of 2-D gels of cytoskeletal preparations from cultures pulse-labeled for $5-10 \mathrm{~min}(a-g)$ or $1 \mathrm{hr}(h)$ and then chased for $0 \mathrm{~min}(a), 25 \mathrm{~min}(b), 55 \mathrm{~min}(c), 115 \mathrm{~min}(d), 235$ $\min (e), 8 \mathrm{hr}(f), 16 \mathrm{hr}(\mathrm{g})$, or $47 \mathrm{hr}(h)$. The appearance of the NF68 (arrowhead) did not detectably change in these pulse-chase experiments. In contrast, the NF145 (large and small arrows) underwent posttranslational modifications that altered its mobility in 2-D gels. Note, however, that the modified forms of NF145 do not appear until at least 30-60 min after its synthesis.

\section{Phosphorylation of newly synthesized NF145 occurs after it is incorporated into the cytoskeleton}

The NF145 of sympathetic neurons can be resolved into four distinct species in 2-D gels. One of these is unphosphorylated, in that it is recognized by antibody to nonphosphorylated NFP (Figs. 3,7 ) and does not comigrate in 2-D gels with NF145 species that can be phosphorylated in situ (Black and Keyser, unpublished observations). This variant apparently corresponds to the initial translation product. The other forms are generated by phosphorylation events (Black and Keyser, unpublished observations) that occur over a period of hours to days after synthesis (Fig. 6). Bennett and DiLullo (1985) have also reported that the posttranslational modification of the 160,000 -molecular-weight NFP of chick neurons occurs over a prolonged time course. Newly synthesized NF145 becomes incorporated into

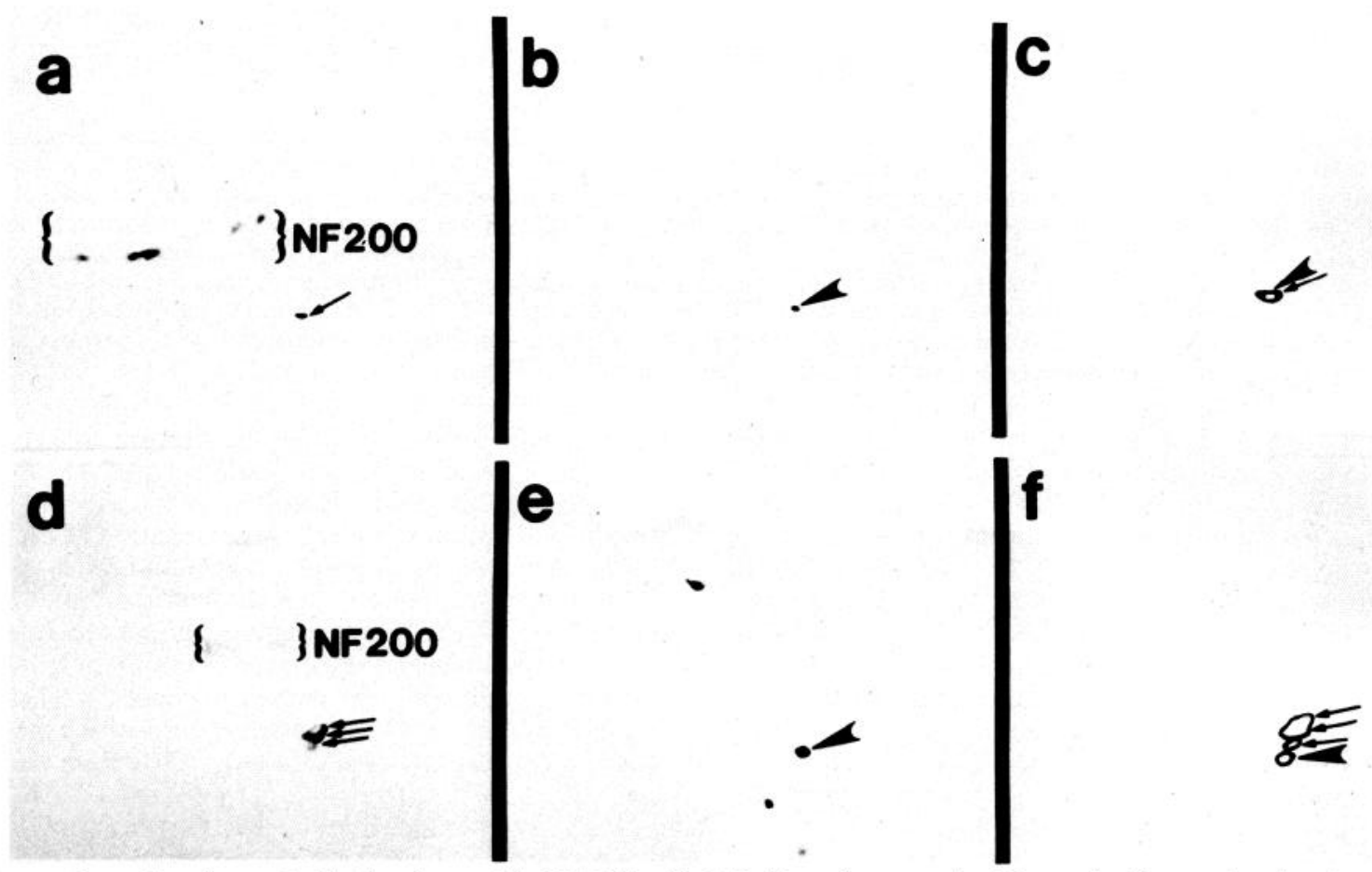

Figure 7. Comparison of newly synthesized and assembled NF145 with NF145 species recognized by antibodies to phosphorylated or nonphosphorylated NFP. Cytoskeletons from cultures pulse-labeled for $5 \mathrm{~min}$ were analyzed on 2-D gels, transferred to nitrocellulose paper, and then probed with antibody to phosphorylated or nonphosphorylated NF145 + NF200. The resulting blots were then incubated with x-ray film to reveal the labeled NF145. Panels $a$ and $d$, immunoblots obtained with antibody to nonphosphorylated or phosphorylated NFP, respectively. Panels $b$ and $e$, autoradiographs of the blots shown in $a$ and $d$, respectively. Panels $c$ and $f$, tracings showing the positions of the immunoreactive (arrows) and labeled (arrowhead) NF145 species. To superimpose the tracings of the blots and the autoradiographs accurately, the perimeter of the blot was spotted with radioactive ink. The labeled ink spots generated spots on the $\mathrm{x}$-ray film. The spots on the film and blot were then used as coordinates to properly line up the autoradiograph on the blot. The coordinate spots are not reproduced in the photograph. Note that the labeled NF145 comigrates with NF145 species recognized by antibody to nonphosphorylated NFP $(c)$, but not with the species recognized by antibodies to phosphorylated NFP $(f)$. 
Table 3. Diffusion of newly synthesized NFP prior to their incorporation into the cytoskeleton

\begin{tabular}{|c|c|c|}
\hline \multirow{2}{*}{$\begin{array}{l}\text { Proportion }(1-\alpha) \\
\text { of newly synthe- } \\
\text { sized NFP } \\
\text { within } x(\mu \mathrm{m}) \\
\text { of the origin } \\
\end{array}$} & \multicolumn{2}{|c|}{ Distance $(x$, in $\mu \mathrm{m})$ from the origin at } \\
\hline & $t=15 \mathrm{~min}$ & $t=30 \mathrm{~min}$ \\
\hline 0.1 & 5 & 8 \\
\hline 0.2 & 11 & 15 \\
\hline 0.3 & 16 & 23 \\
\hline 0.4 & 22 & 31 \\
\hline 0.5 & 29 & 40 \\
\hline 0.6 & 36 & 50 \\
\hline 0.7 & 44 & 62 \\
\hline 0.8 & 54 & 77 \\
\hline 0.9 & 70 & 99 \\
\hline
\end{tabular}

The table presents estimates of the distance (in $\mu \mathrm{m}$ ) that newly synthesized NFP can diffuse from their sites of synthesis (termed the origin) before being incorporated into the cytoskeleton. These estimates assume that the various NFP will diffuse to similar extents because Jacobson and Wojcieszyn (1984) have shown that the diffusion coefficients of proteins injected into cells are independent of their molecular weight (over the range of $12,000-440,000$ ) and Stokes radius (over the range of 16-61 $\AA$ ). From Table 2, newly synthesized NFP is incorporated into the cytoskeleton, to $\sim 90 \%$, within 15 to $30 \mathrm{~min}$ of synthesis. Thus, we have calculated the distances that various proportions of newly synthesized NFP could diffuse in 15 and $30 \mathrm{~min}$. The distance from the origin of a particle undergoing diffusion is a random variable $X$ with a density function $f(x)=(1 / \pi \sigma) \exp \left(-x^{2} / 2 \sigma^{2}\right)$, where $\sigma=$ $(2 D t)^{1 / 2}\left(\mathrm{D}=\right.$ diffusion coefficient $\left[10^{-8} \mathrm{~cm}^{2} / \mathrm{sec}\right]$ and $t=$ time [sec]). This is the density of the absolute value of a normal (Gaussian) variable, with mean $=0$ and $\mathrm{SD}=\sigma$. Let $Z$ be normal with mean $=0$ and $\sigma=1$. We thus have $1-\alpha=P(X \leq$ $x)=P(|Z| \leq x / \sigma)$. This implies that $x / \sigma=z_{\alpha / 2}$ or $x=z_{\alpha / 2} \sigma=z_{\alpha / 2}(2 D t)^{1 / 2}$, where $z_{\alpha / 2}$ is the value, determined from standard tables, such that $P\left(|Z|<z_{\alpha / 2}\right)=1-\alpha$.

the cytoskeleton within minutes of its synthesis (Figs. 4, 5). This time is an order of magnitude shorter than the time required to detect posttranslational modification of the protein (Fig. 6), suggesting that the incorporation of the NF145 into the cytoskeleton occurs prior to its phosphorylation. We have confirmed this possibility by showing that newly synthesized and assembled NF145 consists of a single spot in 2-D gels that comigrates with the NF145 peptide recognized by antibody against nonphosphorylated NF145 (Fig. 7). We conclude that the initial incorporation of the NF145 into the cytoskeleton, presumably into neurofilaments, is not regulated by phosphorylation.

The NF145 contains a core domain that is anchored to the backbone of the filament and a peripheral domain that extends away from the filament backbone (Geisler et al., 1983). The peripheral domain may be involved in the interaction of neurofilaments with each other and with other structures (Geisler et al., 1983; Hirokawa et al., 1984; Willard and Simon, 1981). The peripheral domain contains all of the in vivo phosphorylation sites (Julien and Mushynski, 1983). Thus, phosphorylation of NF145 may be a mechanism for regulating the interaction of NF with other structures.

\section{Spatial interval between the sites of synthesis and} assembly of tubulin and NFP

A major goal of the present studies has been to determine the distance between the sites of synthesis and assembly of cytoskeletal proteins in neurons. This issue relates ultimately to the morphological differentiation of neurons. Neurons consist of several morphologically distinct domains such as the cell body, axon, and dendrites. Several lines of evidence suggest that the morphological differences among these domains are due to underlying differences in the cytoskeletal structures that provide their architectural framework (Caceres et al., 1984; DeCamilli et al., 1984; Drake and Lasek, 1984; Huber and Matus, 1984; Peng et al., 1986; Wuerker and Kirkpatrick, 1972). If this is correct, then the segregation of cytoskeletal components of axons from those of dendrites is an essential aspect of the morphological differentiation of neurons.

Cytoskeletal proteins of axons and dendrites are synthesized in the polysome-containing regions of the neuron, which are located principally in the neuron cell body (Peters et al., 1976). Thus, segregation of cytoskeletal proteins of axons from those of dendrites occurs in the cell body. The basis for this segregation is presently unknown. One model proposes that segregation of axonal and dendritic cytoskeletal proteins is coupled to the assembly of the axonal and dendritic cytoskeletons (Brady and Black, 1985; Lasek, 1981; Lasek and Brady, 1982). One prediction of this model is that the axonal and dendritic cytoskeletons are initially assembled in the cell body. In the present experiments, we have used the time interval between the synthesis and assembly of cytoskeletal proteins to learn about the spatial interval between their sites of synthesis and assembly.

We have estimated the maximal distance between the sites for the synthesis and assembly of NFP by assuming that unpolymerized NFP is freely diffusible in cytoplasm while polymerized NFP is essentially nondiffusible, and that diffusion is the principal force underlying the movement of unpolymerized NFP. In this treatment, the maximum distance between the sites of synthesis and assembly for NFP will correspond to the distance that newly synthesized NFP can diffuse prior to their polymerization. Translational diffusion of NFP in neuronal cytoplasm is unknown. However, diffusion coefficients have been measured for other proteins in other cell types (Kreis et al., 1982; Salmon et al., 1984; Wang et al., 1982; Wojcieszyn et al., 1981 ), and found to range from $10^{-9}$ to $10^{-7} \mathrm{~cm}^{2} / \mathrm{sec}$. We have assumed that the diffusion coefficient of unpolymerized NFP in neuronal cytoplasm will fall within this range. Newly synthesized NFP are $\sim 90 \%$ polymerized within $\sim 30$ min of their synthesis (Table 2). We have estimated how far newly synthesized NFP can diffuse in $30 \mathrm{~min}$, using a diffusion coefficient of $10^{-8}$ $\mathrm{cm}^{2} / \mathrm{sec}$. For these calculations, we have assumed that all of the diffusible NFP are located at a point source (corresponding to the site of synthesis) at $t=0 \mathrm{~min}$, and have used Fick's second law to determine the change in distribution of these proteins due to diffusion after $30 \mathrm{~min}$. Fick's second law is a probability equation in which the distribution of diffusible molecules around a point source at time $t$ is described by a Gaussian distribution. Thus, Fick's second law provides estimates of the distance from a point source within which a given percentage of the diffusible molecules will be found at time $t$. The results of our calculations are given in Table 3.

To evaluate the extent to which newly synthesized NFP is assembled in the cell body, the diffusion distances should be compared to the radius of the cell body, which for the cultured sympathetic neurons used in the present studies is $12-15 \mu \mathrm{m}$. As can be seen, $\sim 20 \%$ of the newly synthesized NFP will be assembled in the neuron cell body. It is not surprising that some newly synthesized NFP is assembled in the cell body because electron-microscopic analyses have revealed numerous neurofilaments therein (Peters et al., 1976; Smith, 1973). However, considerable amounts of the newly synthesized NFP can potentially diffuse for distances that exceed the radius of the cell body before they are incorporated into the cytoskeleton. Thus, at first glance, the calculations suggest that some newly synthesized NFP can diffuse into the neurites prior to their assembly. However, these calculations fail to consider the fact that access into the neurites from the cell body is limited. ${ }^{1}$ We are currently

\footnotetext{
If we assume that the cell body of cultured sympathetic neurons is a sphere with a diameter of $30 \mu \mathrm{m}$, then the surface area $\left(4 \pi r^{2}\right)$ is $2826 \mu \mathrm{m}^{2}$. If the neuron has four neurites, each with a diameter of $1 \mu \mathrm{m}$ at its origin, then the "openings" from the cell body into the neurites will occupy a total area of $3.14 \mu \mathrm{m}^{2}$. This represents only $0.11 \%$ of the total surface area of the cell body. These calculations reveal that access from the cell body into the neurites is quite limited.
} 
developing models that incorporate these additional considerations to more accurately determine the probability that newly synthesized NFP can diffuse into the neurites before they are incorporated into the cytoskeleton. Preliminary indications are that this probability is quite low.

The situation for tubulin probably differs somewhat from that for NFP because the time course of tubulin assembly is slower than that of NFP (compare Figs. 2 and 5, Table 2), and at any given time, $25-30 \%$ of the total tubulin is unassembled (Fig. 2) and presumably able to diffuse in situ (Morris and Lasek, 1984). Clcarly, some polymerization occurs in the cell body because electron-microscopic analyses reveal microtubule-rich domains in the cell body (Lasek and Brady, 1982; Peters et al., 1976; Smith, 1973). Moreover, in view of the apparently limited access from the cell body into the neurites (see above), we propose that it is unlikely that appreciable levels of tubulin can diffuse from the cell body into the neurites. If this is correct, then newly synthesized tubulin is incorporated into the cytoskeleton in the cell body prior to its entry into the neurites.

The kinetic results presented here suggest that the time interval, and by inference the spatial interval, between the synthesis and assembly of tubulin and NFP is relatively short. The primary site for the synthesis of microtubule and NFP is the neuron cell body. Thus, the present results are fully consistent with the hypothesis, originally derived from axonal transport studies (Lasek, 1981), that newly synthesized cytoskeletal proteins are polymerized in the cell body prior to their transport into the neurites. One implication of this hypothesis is that the structure of the axonal and dendritic cytoskeletons is initially elaborated in the neuron cell body.

\section{References}

Bennett, G. S., and C. DiLullo (1985) Slow posttranslational modification of a neurofilament protein. J. Cell Biol. 100: 1799-1804.

Black, M. M., and J. T. Kurdyla (1983a) Microtubule-associated proteins of neurons. J. Cell Biol. 97: 1020-1028.

Black, M. M., and J. T. Kurdyla (1983b) Coupling between the synthesis and assembly of tubulin and neurofilament protein in cultured neurons. J. Cell Biol. 97: 28a.

Black, M. M., and R. J. Lasek (1980) Slow components of axonal transport: Two cytoskeletal networks. J. Cell Biol. 86: 616-623.

Black, M. M., J. M. Cochran, and J. T. Kurdyla (1984) Solubility properties of neuronal tubulin: Evidence for labile and stable microtubules. Brain Res. 295: 255-263.

Blikstad, I., and E. Lazarides (1983) Vimentin filaments are assembled from a soluble precursor in avian erythroid cells. J. Cell Biol. 96: 1803-1808.

Bonner, W. M., and R. A. Laskey (1974) A film detection method for tritium-labeled proteins and nucleic acids in polyacrylamide gels. Eur. J. Biochem. 46: 83-88.

Brady, S. T., and M. M. Black (in press) Axonal transport of microtubule proteins: Cytotypic variation of tubulin and MAPs in neurons. Ann. NY Acad. Sci.

Caceres, A., L. I. Binder, M. R. Payne, P. Bender, L. Rebhun, and O. Steward (1984) Differential subcellular localization of tubulin and the microtubule-associated proteins MAP-2 in brain tissue as revealed by immunocytochemistry with monoclonal hybridoma antibodies. J. Neurosci. 4: 394-410.

DeCamilli, P., P. Miller, F. Navonc, W. E. Theurkauf, and R. B. Vallee (1984) Distribution of microtubule-associated protein 2 (MAP2) in the nervous system of the rat studied by immunofluorescence. Neuroscience $11: 819-846$.

Drake, P., and R. J. Lasek (1984) Regional differences in the neuronal cytoskeleton. J. Neurosci. 4: 1173-1186.

Geisler, N., E. Kaufmann, S. Fischer, U. Plessmann, and K. Weber (1983) Neurofilament architecture combines structural principals of intermediate filaments with carboxy-terminal extensions increasing in size between triplet proteins. EMBO J. 2: 1295-1302.

Hirokawa, N., M. A. Glicksman, and M. B. Willard (1984) Organization of mammalian neurofilament polypeptides within the neuronal cytoskeleton. J. Cell Biol. 99: 1523-1536.

Huber, G., and A. Matus (1984) Differences in the cellular distribution of two microtubule-associated proteins, MAP-1 and MAP-2, in rat brain. J. Neurosci. 4: 151-160.

Jacobson, K., and J. Wojcieszyn (1984) The translational mobility of substances within the cytoplasmic matrix. Proc. Natl. Acad. Sci. USA 81: 6747-6751.

Julien, J-P., and W. E. Mushynski (1982) Multiple phosphorylation sites in mammalian neurofilament polypeptides. J. Biol. Chem. 257: $10467-10470$.

Julien, J-P., and W. E. Mushynski (1983) The distribution of phosphorylation sites among identified proteolytic fragments of mammalian neurofilaments. J. Biol. Chem. 258: 4019-4025.

Kreis, T. E., B. Geiger, and J. Schlessinger (1982) Mobility of microinjected rhodamine actin within living chicken gizzard cells determined by fluorescence photobleaching recovery. Cell 29:835-845.

Lasek, R. J. (1981) The dynamic ordering of neuronal cytoskeletons. Neurosci. Res. Program Bull. 19: 7-32.

Lasek, R. J., and S. T. Brady (1982) The axon: A prototype for studying expressional cytoplasms. Cold Spring Harbor Symp. Quant. Biol. 46: 113-124.

Morris, J. R., and R. J. Lasek (1982) Stable polymers of the axonal cytoskeleton: The axoplasmic ghost. J. Cell Biol. 92: 192-198.

Morris, J. R., and R. J. Lasek (1984) Monomer-polymer equilibria in axon: Direct measurement of tubulin and actin as polymer and monomer in axoplasm. J. Cell Biol. 98: 2064-2076.

Peng, I., L. I. Binder, and M. M. Black (1985) Cultured sympathetic neurons contain a variety of microtubule-associated proteins. Brain Res. 361: 200-211.

Peng, I., L. I. Binder, and M. M. Black (1986) Biochemical and immunological analyses of cytoskeletal domains of neurons. J. Cell Biol. 102: 252-262.

Peters, A., S. S. Palay, and H. deF. Webster (1976) The Fine Structure of the Nervous System, Saunders, Philadelphia.

Salmon, E. D., W. M. Saxton, R. J. Leslie, M. L. Karow, and J. R. McIntosh (1984) Diffusion coefficient of fluorescein-labeled tubulin in the cytoplasm of embryonic cells of a sea urchin: Video image analysis of fluorescence redistribution after photobleaching. J. Cell Biol. 99: 2157-2164.

Schiff, P. B., J. Fant, and S. B. Horowitz (1979) Promotion of microtubule assembly in vitro by taxol. Nature 277: 665-667.

Schliwa, M., U. Euteneuer, J. Chloe Bulinski, and J. G. Izant (1981) Calcium lability of cytoplasmic microtubules and its modulation by microtubule associated proteins. Proc. Natl. Acad. Sci. USA 78: 10371041.

Smith, D. E. (1973) The location of neurofilaments and microtubules during the postnatal development of Clark's nucleus in the kitten. Brain Res. 55: 41-53.

Wang, Y.-L., F. Lanni, P. L. McNeil, B. R. Ware, and D. L. Taylor (1982) Mobility of cytoplasmic actin and membrane-associated actin in living cells. Proc. Natl. Acad. Sci. USA 79: 4660-4664.

Weiss, P., and H. Wang (1936) Neurofibrils in dorsal root ganglion cells of the chick cultivated in vitro. Anat. Rec. 67:105-117.

Willard, M., and C. Simon (1981) Antibody decoration of neurofilaments. J. Cell Biol. 89: 198-205.

Wojcieszyn, J. W., R. A. Schlcgel, E.-S. Wu, and K. A. Jacobson (1981) Diffusion of injected macromolecules within the cytoplasm of living cells. Proc. Natl. Acad. Sci. USA 78: 4407-4410.

Wong, J., S. B. Hutchinson, and R. K. H. Liem (1984) An isoelectric variant of the 150,000 -dalton neurofilament polypeptide. Evidence that phosphorylation state affects its association with the filament. J. Biol. Chem. 259: 10867-10874.

Wuerker, R., and J. Kirkpatrick (1972) Neuronal microtubules, neurofilaments and microfilaments. Int. Rev. Cytol. 33: 43-75. 